\title{
NURBS on Criss-cross Triangulations and Applications
}

\author{
Isabella Cravero, Catterina Dagnino and Sara Remogna* \\ Department of Mathematics, University of Torino, via C. Alberto, 10 - 10123 Torino, Italy \\ Email: sara.remogna@unito.it
}

\begin{abstract}
In this paper we consider and analyse NURBS based on bivariate quadratic B-splines on criss-cross triangulations of the parametric domain $\Omega_{0}=[0,1] \times[0,1]$, presenting their main properties, showing their performances to exactly construct quadric surfaces and reporting some applications related to the modeling of objects. Moreover, we propose applications to the numerical solution of partial differential equations, with mixed boundary conditions on a given physical domain $\Omega$, by using three different spline methods to set the prescribed Dirichlet boundary conditions.
\end{abstract}

Keywords: Bivariate NURBS, criss-cross triangulation, differential problem.

\section{Introduction}

In this paper, we consider and analyse NURBS, based on bivariate quadratic B-splines on criss-cross triangulations of the parametric domain $\Omega_{0}=[0,1] \times[0,1]$, introduced in [1]. Since the above B-splines are of total degree, the bivariate spline functions based on them avoid possible unwanted oscillations and inflection points, as noted in [1]. Moreover, they are those of the lowest degree having continuous first partial derivatives and they allow the control of smoothness across directions not only parallel to the coordinate axes.

We underline that the space of quadratic splines on criss-cross triangulations (see e.g. $[2,3,4,5,6,7,8,9]$ ) has been considered in the solution of several kinds of problems, like construction of approximation operators with special properties (see e.g. $[10,11,12,13,14,15,16]$ ), solution of integral equations (see e.g. [17]), numerical integration (see e.g. [18]), surface reconstruction (see e.g. $[1,19,20,21,22]$ ). Here we want to give a further contribution to the researches on this topic.

After presenting the main performances of the above NURBS, we propose applications to the solution of partial differential equations with mixed boundary conditions on a given physical domain $\Omega$. Since many domains of interest in applications are often described by conic sections, then we represent them by such NURBS. Furthermore, in order to avoid the heavy computations related to their derivatives and integrals, since the computation with B-splines is strictly related to the corresponding NURBS, we use the same above B-splines to get the basis for the solution space of the differential problem. In this way, we keep a unique description of the geometry, while avoiding the use of rational functions in the discretization of the solution.

Here is an outline of the paper. In Section 2, we report definitions and present properties of bivariate quadratic spline spaces on criss-cross triangulations of the parametric domain, introducing B-spline basis. In Section 3 we construct NURBS based on such B-splines, present their main properties, show their performances to exactly represent quadric surfaces and report some applications related to the modeling of objects. In Section 4, we propose applications to the solution of diffusion problems with mixed boundary conditions on the physical domain $\Omega$. In particular, by the above NURBS we generate surfaces reproducing $\Omega$ and we use the same B-splines to get a basis for the solution space, considering several spline approximation schemes to impose non-homogeneous Dirichlet boundary conditions. Finally, some numerical examples are provided, with comparisons with other classical methods based on finite elements and/or biquadratic B-splines.

\section{Bivariate Quadratic Spline Spaces on Criss-cross Triangulations}

In this section we present bivariate quadratic spline spaces on criss-cross triangulations and their properties. 


\subsection{The Spline Space $\mathcal{S}_{2}^{1}\left(\mathcal{T}_{m n}\right)$}

Given two positive integers $m$ and $n$, let $\Omega_{0}:=\left\{(\xi, \eta) \in \mathbb{R}^{2}: 0 \leq \xi, \eta \leq 1\right\}$ be the unit square decomposed into $m n$ subrectangles by the two non uniform partitions $\bar{\xi}:=\left\{\xi_{i}, 0 \leq i \leq m\right\}, \bar{\eta}:=\left\{\eta_{j}, 0 \leq j \leq n\right\}$ of the segments $\left[\xi_{0}, \xi_{m}\right]=[0,1]$ and $\left[\eta_{0}, \eta_{n}\right]=[0,1]$, respectively.

We denote by $\mathcal{R}_{m n}$ the rectangular partition of $\Omega_{0}$ given by $\bar{\xi} \times \bar{\eta}$ and by $\mathcal{S}_{2,2}^{1,1}\left(\mathcal{R}_{m n}\right)$ the space of $C^{1}$ biquadratic tensor product splines.

Now, let $\mathcal{T}_{m n}$ be the criss-cross triangulation of $\Omega_{0}$, defined by drawing the two diagonals in each subrectangle. We consider the space

$$
\mathcal{S}_{2}^{1}\left(\mathcal{T}_{m n}\right):=\left\{s \in C^{1}\left(\Omega_{0}\right):\left.s\right|_{T} \in \mathbb{P}_{2}, \text { for each triangular cell } T \text { of } \mathcal{T}_{m n}\right\},
$$

whose dimension is

$$
N_{h}=(m+2)(n+2)-1
$$

where $\mathbb{P}_{\ell}$ is the space of bivariate polynomials of total degree less than or equal to $\ell$ [4, Sect. 2.5].

\subsection{Spanning Set, Basis and Properties of $\mathcal{S}_{2}^{1}\left(\mathcal{T}_{m n}\right)$}

Setting $\mathcal{K}_{m n}:=\{(i, j): 0 \leq i \leq m+1,0 \leq j \leq n+1\}$, let

$$
\mathcal{B}_{m n}:=\left\{B_{i j},(i, j) \in \mathcal{K}_{m n}\right\}
$$

be the collection of $(m+2)(n+2)$ B-splines spanning the space $\mathcal{S}_{2}^{1}\left(\mathcal{T}_{m n}\right)$ [7], with knots

$$
\begin{aligned}
& \xi_{-2}=\xi_{-1}=0=\xi_{0}<\xi_{1}<\ldots<\xi_{m}=1=\xi_{m+1}=\xi_{m+2} \\
& \eta_{-2}=\eta_{-1}=0=\eta_{0}<\eta_{1}<\ldots<\eta_{n}=1=\eta_{n+1}=\eta_{n+2} .
\end{aligned}
$$

In $\mathcal{B}_{m n}$, we can distinguish three kinds of B-splines. There are:

- a first-boundary-layer of $2 m+2 n+4$ B-splines, with triple knots on their support, whose restrictions to the boundary $\partial \Omega_{0}$ of $\Omega_{0}$ are univariate quadratic B-splines, i.e. $B_{i j},(i, j) \in \widetilde{\mathcal{K}}_{m n}$, with $\widetilde{\mathcal{K}}_{m n}:=$ $\{(i, 0),(i, n+1), 0 \leq i \leq m+1 ;(0, j),(m+1, j), 1 \leq j \leq n\}$;

- a second-boundary-layer of $2 m+2 n-4$ B-splines, with double knots on their support, i.e. $B_{i 1}, B_{i n}$, $1 \leq i \leq m, B_{1 j}, B_{m j}, 2 \leq j \leq n-1$;

- $(m-2)(n-2)$ inner B-splines, with simple knots, i.e. $B_{i j}, 2 \leq i \leq m-1,2 \leq j \leq n-1$.

We remark that the first-boundary-layer and the second-boundary-layer B-splines can be expressed as linear combination of classical B-splines with octagonal support and simple knots [3,23].

Therefore, an element $s \in \mathcal{S}_{2}^{1}\left(\mathcal{T}_{m n}\right)$ can be written in the form

$$
s(\xi, \eta)=\sum_{i=0}^{m+1} \sum_{j=0}^{n+1} \alpha_{i j} B_{i j}(\xi, \eta), \quad \alpha_{i j} \in \mathbb{R},(\xi, \eta) \in \Omega_{0} .
$$

Since the knot multiplicity affects the B-spline smoothness, i.e. $B_{i j}$ is $2-r$ differentiable, if $r$ is the knot multiplicity, the first-boundary-layer B-splines have a jump on $\partial \Omega_{0}$, the second-boundary-layer ones are $C^{0}$ on $\partial \Omega_{0}$ and the inner ones are $C^{1}$ everywhere.

We recall $[2,3,24]$ that the second-boundary-layer B-splines and the inner ones coincide with the so called "interior", "side" and "corner" B-splines, spanning the space of $C^{1}$ quadratic piecewise polynomials with boundary conditions. In Fig. 1 we show some B-splines of $\mathcal{B}_{m n}$. Moreover, in the case of uniform knot partition, i.e $\xi_{i}-\xi_{i-1}=\eta_{j}-\eta_{j-1}=h, i=1, \ldots, m+1, j=1, \ldots, n+1$, the inner B-splines are the classical box splines (see e.g. [4, Sect. 2.4], [5], [25, pag. 5], [26, Sect. 12.2] and the references therein).

In order to evaluate the B-splines in (2) (or their derivatives), it is convenient to consider their B-form, i.e. their expression by means of the Bernstein-Bézier coefficients (BB-coefficients) and then apply the de Casteljau algorithm for triangular surfaces [26, Sect. 2.5]. Since each B-spline is a polynomial of total degree two in each triangle of $\mathcal{T}_{m n}$, it is described by six BB-coefficients, ensuring the $C^{1}$ smoothness. 


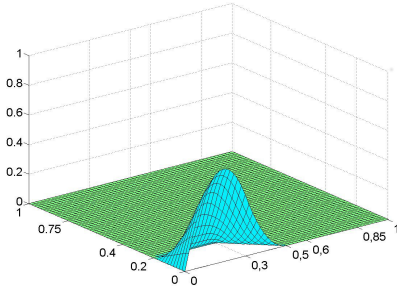

(a)

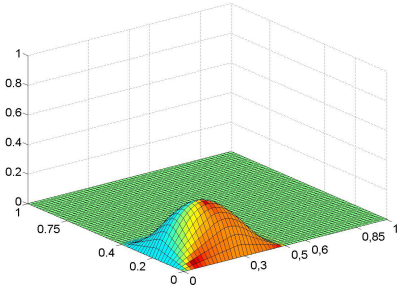

(b)

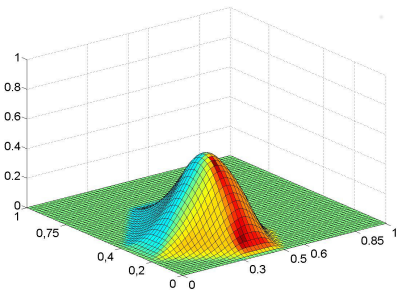

(c)

Figure 1. The graph of $(a)$ the first-boundary-layer B-spline $B_{10} ;(b)$ the second-boundary-layer B-spline $B_{11} ;(c)$ the inner B-spline $B_{22}$, in $\mathcal{S}_{2}^{1}\left(\mathcal{T}_{54}\right)$, with $\bar{\xi}=\{0,0.3,0.5,0.6,0.85,1\}$ and $\bar{\eta}=\{0,0.2,0.4,0.75,1\}$.

Consequently, its first partial derivatives are polynomials of total degree one in such triangles, where they are described by three BB-coefficients, ensuring the $C^{0}$ smoothness.

The BB-coefficients of the inner B-splines $\left\{B_{i j}, 2 \leq i \leq m-1,2 \leq j \leq n-1\right\}$ are given in [27] and are reported in Fig. 2, the other ones can be found in $[5,6]$. In particular we recall that the BB-coefficients of any $B_{i j}$ are obtained by those reported in Fig. 2, conveniently setting $h_{p}=\xi_{p}-\xi_{p-1}, p=i-1, i, i+1$, and/or $k_{q}=\eta_{q}-\eta_{q-1}, q=j-1, j, j+1$, equal to zero, in the case of double or triple knots. When $\frac{0}{0}$ occurs, we set the corresponding value equal to zero.

Concerning the properties of the B-splines belonging to $\mathcal{B}_{m n}$, we recall that they are positive and form a partition of unity. Moreover, the first-boundary-layer B-splines are linearly independent as the univariate ones, while the second-boundary-layer and the inner B-splines are linearly dependent. In order to obtain a B-spline basis for $\mathcal{S}_{2}^{1}\left(\mathcal{T}_{m n}\right)$ we have to neglect one B-spline from the spanning set $\mathcal{B}_{m n}$, getting the set

$$
\mathcal{B}:=\left\{B_{i j},(i, j) \in \overline{\mathcal{K}}_{m n}\right\} \subset \mathcal{B}_{m n},
$$

where $\overline{\mathcal{K}}_{m n}:=\left\{(i, j) \in \mathcal{K}_{m n},(i, j) \neq\left(i_{0}, j_{0}\right)\right\}$, for any pair of indices $\left(i_{0}, j_{0}\right) \in \widehat{\mathcal{K}}_{m n}[4$, Sect. 2.7], [7], with $\widehat{\mathcal{K}}_{m n}:=\{(i, j), 1 \leq i \leq m, 1 \leq j \leq n\}$. We remark that the cardinality of $\mathcal{B}$ is equal to $N_{h}$, with $N_{h}$ defined in (1).

Moreover, we underline that in [8] the refinement equation for quadratic B-splines is proposed. In particular, considering the subtriangulation $\mathcal{T}_{2 m, 2 n}$ of $\mathcal{T}_{m n}$, obtained by subdividing each rectangle $\left[\xi_{i}, \xi_{i+1}\right] \times\left[\eta_{j}, \eta_{j+1}\right]$ into four equal subrectangles and then by drawing the two diagonals in each subrectangle, since $\mathcal{S}_{2}^{1}\left(\mathcal{T}_{m n}\right) \subset \mathcal{S}_{2}^{1}\left(\mathcal{T}_{2 m, 2 n}\right)$, each coarse B-spline (i.e. belonging to $\mathcal{S}_{2}^{1}\left(\mathcal{T}_{m n}\right)$ ) is expressed as linear combination of fine B-splines (i.e. belonging to $\mathcal{S}_{2}^{1}\left(\mathcal{T}_{2 m, 2 n}\right)$ ).

Furthermore, given a function $\rho \in C\left(\Omega_{0}\right)$, we can establish how well a spline in $\mathcal{S}_{2}^{1}\left(\mathcal{T}_{m n}\right)$ is able to approximate such a function. Indeed, concerning the approximation properties of bivariate $C^{1}$ quadratic spline spaces on criss-cross triangulations, we can refer to [9], where the authors give error bounds for functions and partial derivatives in terms of the smoothness of functions that are approximated and the characteristics of the triangulation. In particular, since we know that there exist quasi-interpolating operators exact on the space $\mathbb{P}_{2}$ (see e.g [4, Sect. 2.4], $[7,9,19]$ ), then the optimal approximation order is achieved for sufficiently smooth functions, i.e. if $\rho \in C^{3}\left(\Omega_{0}\right)$ there exists a constant $C>0$ such that

$$
\inf _{s \in \mathcal{S}_{2}^{1}\left(\mathcal{T}_{m n}\right)}\|\rho-s\|_{\infty} \leq C \Delta^{3} \max \left\{\left\|D^{\alpha_{1}, \alpha_{2}} \rho\right\|_{\infty}: \alpha_{1}+\alpha_{2}=3\right\}
$$

where $\Delta:=\max \left\{\operatorname{diam}(T) \mid T\right.$ is a triangle of $\left.\mathcal{T}_{m n}\right\}$.

An interesting property of the spline functions in $\mathcal{S}_{2}^{1}\left(\mathcal{T}_{m n}\right)$ is the control of $C^{1}$ smoothness across directions not only parallel to the coordinate axes.

We highlight it by the following example, where we consider the function $\rho(\xi, \eta)=(3 \eta-2 \xi+1) \mid 3 \eta-$ $2 \xi+1 \mid,(\xi, \eta) \in \Omega_{0}$, that is $C^{1}$ across the line $3 \eta-2 \xi+1=0$, but it has a discontinuity of the second directional derivative in the direction $\left[\frac{2 \sqrt{13}}{13},-\frac{3 \sqrt{13}}{13}\right]^{T}$ is across the same above line. 


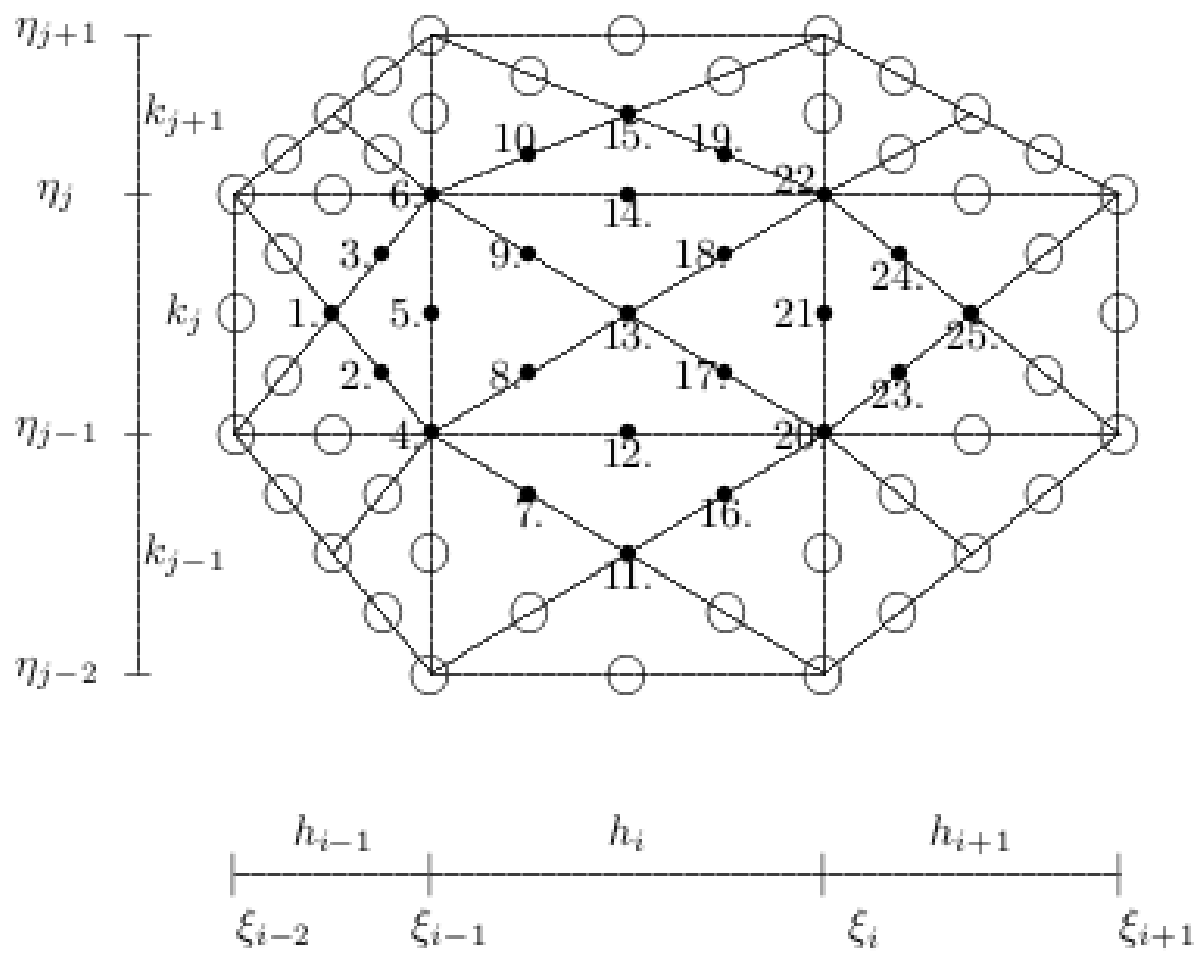

(a)
1. $\frac{\sigma_{i}^{\prime}}{4}$,
2. $\frac{\sigma_{i}^{\prime}}{2}$
3. $\frac{\sigma_{i}^{\prime}}{2}$,
4. $\sigma_{i}^{\prime} \tau_{j}^{\prime}$
5. $\sigma_{i}^{\prime}$,
6. $\sigma_{i}^{\prime} \tau_{j+1}, 7 \cdot \frac{\tau_{j}^{\prime}}{2}$,
8. $\frac{\sigma_{i}^{\prime}+\tau_{j}^{\prime}}{2}$
9. $\frac{\sigma_{i}^{\prime}+\tau_{j+1}}{2}, 10 . \frac{\tau_{j+1}}{2}$,
11. $\frac{\tau_{j}^{\prime}}{4}$,
12. $\tau_{j}^{\prime}$,
13. $\frac{\sigma_{i}^{\prime}+\sigma_{i+1}+\tau^{\prime}}{4}$ 18. $\frac{\sigma_{i+1}+\tau_{j+1}}{2}$,
14. $\tau_{j+1}$
15. $\frac{\tau_{j+1}}{4}$,
16. $\frac{\tau_{j}^{\prime}}{2}$,
17. $\frac{\sigma_{i+1}+\tau_{j}^{\prime}}{2}$,
21. $\sigma_{i+1}, 22 . \sigma_{i+1} \tau_{j+1}$, 23. $\frac{\sigma_{i+1}}{2}$,
19. $\frac{\tau_{j+1}}{2}$,
20. $\sigma_{i+1} \tau_{j}^{\prime}$,
24. $\frac{\sigma_{i+1}}{2}$,
25. $\frac{\sigma_{i+1}}{4}$,

(b)

Figure 2. (a) Support and (b) BB-coefficients of the $C^{1}$ B-splines $B_{i j}, 2 \leq i \leq m-1,2 \leq j \leq n-1$, where "O" denotes a zero B-coefficient and $\sigma_{i+1}=\frac{h_{i+1}}{h_{i}+h_{i+1}}, \sigma_{i}^{\prime}=\frac{h_{i-1}}{h_{i-1}+h_{i}}, \tau_{j+1}=\frac{k_{j+1}}{k_{j}+k_{j+1}}, \tau_{j}^{\prime}=\frac{k_{j-1}}{k_{j-1}+k_{j}}$, with $h_{i}=\xi_{i}-\xi_{i-1}$, $k_{j}=\eta_{j}-\eta_{j-1}$. with

We assume $\bar{\xi}=\{0,1 / 2,1\}, \bar{\eta}=\{0,1 / 3,1\}$ as knot vectors and we set $\alpha_{i j}=\rho\left(s_{i}, t_{j}\right), i, j=0, \ldots, 3$,

$$
s_{i}:=\frac{\xi_{i-1}+\xi_{i}}{2}, \quad t_{j}:=\frac{\eta_{j-1}+\eta_{j}}{2} .
$$

Then, we approximate the function $\rho$ by the bivariate spline $s$ belonging to $\mathcal{S}_{2}^{1}\left(\mathcal{T}_{22}\right)$ and of the form (4). By using the above knot vectors and coefficients $\alpha_{i j}$, we also construct the approximation of $\rho$ in the space $\mathcal{S}_{2,2}^{1,1}\left(\mathcal{R}_{22}\right)$. In this case the blending functions are the biquadratic B-splines on $\bar{\xi} \times \bar{\eta}$. We remark that the spline $s$ is defined by the well known bivariate variation diminishing operator (see e.g. [4, Sect. 2.4], [7]) and the spline belonging to $\mathcal{S}_{2,2}^{1,1}\left(\mathcal{R}_{22}\right)$ is the tensor product of two univariate spline quasi-interpolating variation diminishing schemes [28, Chaps. XII, XVII].

Then we construct their first directional derivatives across the line $3 \eta-2 \xi+1=0$. 
In Figs. 3(a) and 3(b) we report the graphs of the approximate first directional derivatives of $\rho$ in the direction $\left[\frac{2 \sqrt{13}}{13},-\frac{3 \sqrt{13}}{13}\right]^{T}$, computed in the two above spline spaces, respectively. In $(a)$ we can notice the exact reconstruction of the $C^{0}$ continuity of first directional derivative of $\rho$, by using the spline belonging to $\mathcal{S}_{2}^{1}\left(\mathcal{T}_{22}\right)$, while in $(b)$ the first directional derivative of the spline belonging to $\mathcal{S}_{2,2}^{1,1}\left(\mathcal{R}_{22}\right)$ does not reproduce the requested $C^{0}$ smoothness across the line $3 \eta-2 \xi+1=0$.

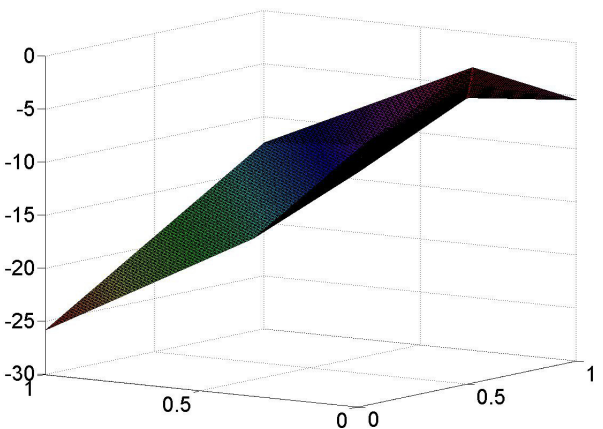

(a)

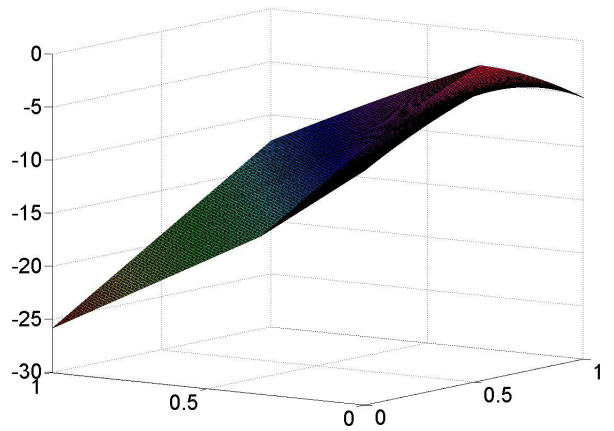

(b)

Figure 3. The graphs of the approximate first directional derivatives of $\rho(\xi, \eta)=(3 \eta-2 \xi+1)|3 \eta-2 \xi+1|$ in the direction $\left[\frac{2 \sqrt{13}}{13},-\frac{3 \sqrt{13}}{13}\right]^{T}(a)$ in $\mathcal{S}_{2}^{1}\left(\mathcal{T}_{22}\right)$ and $(b)$ in $\mathcal{S}_{2,2}^{1,1}\left(\mathcal{R}_{22}\right)$.

\subsection{Splines on the Boundary of $\Omega_{0}$}

We consider the boundary $\partial \Omega_{0}$ of $\Omega_{0}$ and we remark that the restrictions of first-boundary-layer B-splines to $\partial \Omega_{0}$ are univariate B-splines. If we focus, without loss of generality, on the edge $[\xi, 0]$ of $\Omega_{0}$, the B-splines belonging to $\mathcal{B}_{m n}$, different from zero on $[\xi, 0]$ are the $B_{i 0}$ 's, $i=0, \ldots, m+1$ and their restrictions to $[\xi, 0]$ coincide with the univariate $C^{1}$ quadratic B-splines on the knot partition (3). Thus the spline $s$ in (4), restricted to $\partial \Omega_{0}$, can be expressed in the form

$$
s(\xi, 0)=\sum_{i=0}^{m+1} \alpha_{i 0} B_{i 0}(\xi, 0) .
$$

Being interested in the approximation of $\left.\rho\right|_{[\xi, 0]}=\rho(\xi, 0)=: \omega(\xi)$, the coefficients $\alpha_{i 0}$ 's in $(7)$ can be obtained by using different univariate spline schemes. Next we show three possible choices.

(i) If we set $\alpha_{i 0}=\omega\left(s_{i}\right), i=0, \ldots, m+1$, where the points $s_{i}, i=0, \ldots, m+1$, are the Greville abscissas defined in (6), we get the simplest choice and it yields the well-known univariate Schoenberg-Marsden operator $S_{1}$, exact on the space of linear polynomials (see e.g. [28, pag. 141]). Therefore, from results known in the literature, we know that the univariate operator $S_{1}$ is near optimal, i.e. for a function $\omega \in C^{2}(0,1)$, there exists a constant $C_{1}>0$ such that

$$
\left\|\omega-S_{1} \omega\right\|_{\infty} \leq C_{1} h^{2}\left\|\omega^{\prime \prime}\right\|_{\infty}
$$

where $S_{1} \omega(\xi)=\sum_{i=0}^{m+1} \omega\left(s_{i}\right) B_{i 0}(\xi, 0)$ and

$$
h=\max _{i=1, \ldots m}\left(\xi_{i}-\xi_{i-1}\right) .
$$


(ii) We can set the $\alpha_{i 0}$ 's in (7) as the coefficients of the univariate quadratic spline quasi-interpolant $S_{2}$, defined and studied in [7], i.e. $\alpha_{i 0}=\mu_{i}(\omega)$, where

$$
\begin{aligned}
& \mu_{0}(\omega)=\omega\left(s_{0}\right), \\
& \mu_{i}(\omega)=a_{i} \omega\left(s_{i-1}\right)+b_{i} \omega\left(s_{i}\right)+c_{i} \omega\left(s_{i+1}\right), i=1, \ldots, m, \\
& \mu_{m+1}(\omega)=\omega\left(s_{m+1}\right)
\end{aligned}
$$

with $s_{i}$ defined in (6) and

$$
\begin{gathered}
a_{i}=-\frac{\sigma_{i}^{2} \sigma_{i+1}^{\prime}}{\sigma_{i}+\sigma_{i+1}^{\prime}}, \quad b_{i}=1+\sigma_{i} \sigma_{i+1}^{\prime}, \quad c_{i}=-\frac{\sigma_{i}\left(\sigma_{i+1}^{\prime}\right)^{2}}{\sigma_{i}+\sigma_{i+1}^{\prime}} \\
\sigma_{i}=\frac{h_{i}}{h_{i-1}+h_{i}} \quad \sigma_{i}^{\prime}=\frac{h_{i-1}}{h_{i-1}+h_{i}}=1-\sigma_{i} \\
h_{i}=\xi_{i}-\xi_{i-1}, \quad i=0, \ldots, m+1 .
\end{gathered}
$$

In this case we have a univariate operator exact on the space of quadratic polynomials. Therefore, from results known in the literature, such a univariate quasi-interpolant achieves the optimal approximation order for sufficiently smooth functions, i.e. for $\omega \in C^{3}(0,1)$, there exists a constant $C_{2}>0$ such that

$$
\left\|\omega-S_{2} \omega\right\|_{\infty} \leq C_{2} h^{3}\left\|\omega^{(3)}\right\|_{\infty}
$$

with $h$ defined in (8).

(iii) Finally, we can choose the $\alpha_{i 0}$ 's in (7) as the solution of the following interpolation problem

$$
s\left(s_{i}, 0\right)=\omega\left(s_{i}\right), i=0, \ldots, m+1,
$$

with $s_{i}, i=0, \ldots, m+1$, defined in (6). It is well-known that the linear system

$$
\sum_{i=0}^{m+1} \alpha_{i 0} B_{i 0}\left(s_{i}, 0\right)=\omega\left(s_{i}\right), i=0, \ldots, m+1
$$

has a unique solution and we have the optimal approximation order three for sufficiently smooth functions (see [28, Chap. XIII]).

\subsection{Generalization to Unequally Smooth Bivariate Quadratic Spline Spaces on Criss-cross Triangulations}

A generalization of the space $\mathcal{S}_{2}^{1}\left(\mathcal{T}_{m n}\right)$ is the space $\mathcal{S}_{2}^{\left(\bar{\mu}^{\xi}, \bar{\mu}^{\eta}\right)}\left(\mathcal{T}_{m n}\right)$ of unequally smooth bivariate quadratic piecewise polynomials on $\mathcal{T}_{m n}$ (see e.g. [1,29]), where $\bar{\mu}^{\xi}=\left(\mu_{i}^{\xi}\right)_{i=1}^{m-1}, \bar{\mu}^{\eta}=\left(\mu_{j}^{\eta}\right)_{j=1}^{n-1}$ are vectors whose elements can be 1, 0, -1 and denote the $C^{1}, C^{0}, C^{-1}$ spline smoothness, respectively, across the inner grid lines $\xi-\xi_{i}=0, i=1, \ldots, m-1$ and $\eta-\eta_{j}=0, j=1, \ldots, n-1$, while the spline smoothness across all oblique mesh segments ${ }^{1}$ is $C^{1}$.

In the case of jumps at $\xi=\xi_{i}$ and/or $\eta=\eta_{j}$, in order to uniquely define $s \in \mathcal{S}_{2}^{\left(\bar{\mu}^{\xi}, \bar{\mu}^{\eta}\right)}\left(\mathcal{T}_{m n}\right)$, we set

$$
s\left(\xi_{i}, \eta\right)=\left\{\begin{array}{l}
s\left(\xi_{i}^{+}, \eta\right), i=0, \ldots, m-1, \\
s\left(\xi_{i}^{-}, \eta\right), i=m,
\end{array}, \quad s\left(\xi, \eta_{j}\right)=\left\{\begin{array}{l}
s\left(\xi, \eta_{j}^{+}\right), j=0, \ldots, n-1, \\
s\left(\xi, \eta_{j}^{-}\right), j=n .
\end{array}\right.\right.
$$

The properties of such spline spaces are studied in [29]. In particular, we recall that the dimension of $\mathcal{S}_{2}^{\left(\bar{\mu}^{\xi}, \bar{\mu}^{\eta}\right)}\left(\mathcal{T}_{m n}\right)$ is expressed by a formula depending on $m, n$ and the required smoothness.

Moreover, the problem of the construction of a basis for the space $\mathcal{S}_{2}^{\left(\bar{\mu}^{\xi}, \bar{\mu}^{\eta}\right)}\left(\mathcal{T}_{m n}\right)$ is faced up by generating a spanning set and then extracting a basis. First of all, we observe that we can associate a knot multiplicity with the required smoothness, i.e. for $i=1, \ldots, m-1$, the number $\xi_{i}$ occurs exactly

\footnotetext{
${ }^{1}$ According to [4], we call mesh segments the line segments that form the boundary of each triangular cell of $\mathcal{T}_{m n}$.
} 
$2-\mu_{i}^{\xi}$ times and for $j=1, \ldots, n-1$, the number $\eta_{j}$ occurs exactly $2-\mu_{j}^{\eta}$ times. Then, the system of generators is created in the same way explained in Section 2.2, i.e. considering the BB-coefficients of the inner B-spline reported in Fig. 2, conveniently setting the steplengths equal to zero, in case of double or triple knots [30]. Once constructed the spanning set, then we can obtain the B-spline basis, by conveniently deleting a certain number of generators, depending on the number of spline unequally smooth conditions.

\section{Construction of Bivariate Quadratic NURBS with Applications to the Modeling of Objects.}

In this section we construct bivariate quadratic NURBS surfaces based on the B-splines of Section 2.

Quadratic NURBS functions on $\mathcal{T}_{m n}$ can be obtained from the previous B-spline functions. Let $\left\{w_{i j}\right\}_{(i, j) \in \mathcal{K}_{m n}}$ be a set of positive weights, from (2) we can define the set of quadratic NURBS functions on $\mathcal{T}_{m n}$

$$
\mathcal{N}_{m n}:=\left\{R_{i j}(\xi, \eta)=\frac{w_{i j} B_{i j}(\xi, \eta)}{\sum_{(\alpha, \beta) \in \mathcal{K}_{m n}} w_{\alpha \beta} B_{\alpha \beta}(\xi, \eta)},(i, j) \in \mathcal{K}_{m n}\right\},
$$

spanning the space of quadratic rational splines on $\mathcal{T}_{m n}[1]$.

Therefore, a quadratic NURBS surface has the form

$$
\mathbf{S}(\xi, \eta)=\sum_{(i, j) \in \mathcal{K}_{m n}} \mathbf{P}_{i j} R_{i j}(\xi, \eta), \quad(\xi, \eta) \in \Omega_{0},
$$

where $\left(\mathbf{P}_{i j}\right)_{(i, j) \in \mathcal{K}_{m n}}$ is a given net of control points in $\mathbb{R}^{3}$. In order to generate the surface $\mathbf{S}(\xi, \eta)$, we have constructed a procedure based on the homogeneous coordinate representation of a NURBS surface in the three-dimensional space as a B-spline surface in the four-dimensional space. The use of homogeneous coordinate representations to handle NURBS surfaces is usual in literature (see e.g. [31, Chap. 4]).

We assume $p_{i j}=\left(s_{i}, t_{j}\right) \in \Omega_{0}$ as the pre-image of $\mathbf{P}_{i j}$, with $s_{i}$ and $t_{j}$ defined in (6).

We remark that, in the case $w_{i j}=w$, for all $(i, j)$, then $R_{i j}=B_{i j} \in \mathcal{B}_{m n}$ and therefore $\mathbf{S}(\xi, \eta)$ is a bivariate quadratic B-spline surface. If, in addition, we consider a functional parametrization, $\mathbf{S}(\xi, \eta)$ is the spline function defined by the well known bivariate "variation diminishing" operator, reproducing bilinear functions.

The surface (10) has both the convex hull property and the affine transformation invariance one. Moreover, from the B-spline locality property, the surface interpolates the control points whose pre-images are the corner points of $\Omega_{0}$.

The introduction of rational blending functions allows us to exactly model a larger number of shapes. For example if we consider the knot vectors $\bar{\xi}=\bar{\eta}=\{0,1\}$, the control points and weights given in Table 1, we obtain the quadratic NURBS surface shown in Fig. 4(a). According to the properties of rational quadratic Bézier curves, the four boundary curves are quarter circles.

Moreover, in Fig. 4(b), we show the NURBS surface based on $C^{1}$ biquadratic B-splines and constructed by using the same knot vectors $\bar{\xi}=\bar{\eta}=\{0,1\}$, control points and weights given in Table 1 . We can notice the different behaviour of the two surfaces in Fig. 4(a) and (b), that is, as noted in [1], tensor product splines (see Fig. 4(b)) may have some inflection points due to their higher coordinate degree four with respect to bivariate spline functions having total degree two (see Fig. 4(a)).

Table 1. Control points and weights.

\begin{tabular}{|c|ccc|ccc|}
\hline$i$ & $\mathbf{P}_{i 0}$ & $\mathbf{P}_{i 1}$ & $\mathbf{P}_{i 2}$ & $w_{i 0}$ & $w_{i 1}$ & $w_{i 2}$ \\
\hline 0 & $(-1,1,0)$ & $(0,1,1)$ & $(1,1,0)$ & 1 & $\frac{\sqrt{2}}{2}$ & 1 \\
1 & $(-1,0,1)$ & $(0,0,-1)$ & $(1,0,1)$ & $\frac{\sqrt{2}}{2}$ & 1 & $\frac{\sqrt{2}}{2}$ \\
2 & $(-1,-1,0)$ & $(0,-1,1)$ & $(1,-1,0)$ & 1 & $\frac{\sqrt{2}}{2}$ & 1 \\
\hline
\end{tabular}




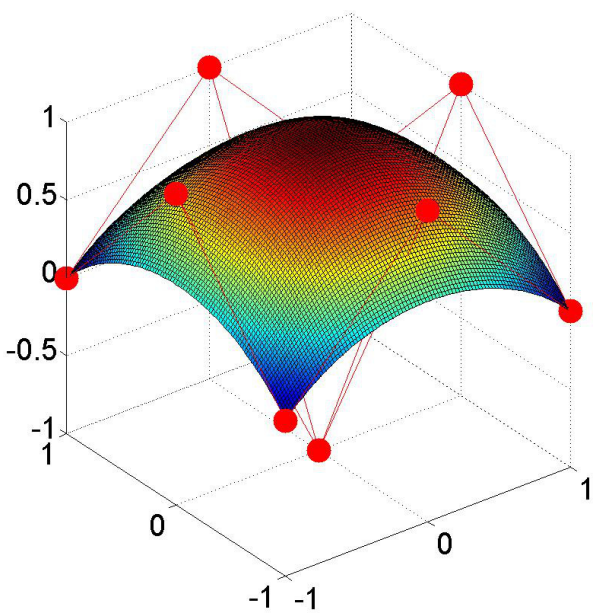

(a)

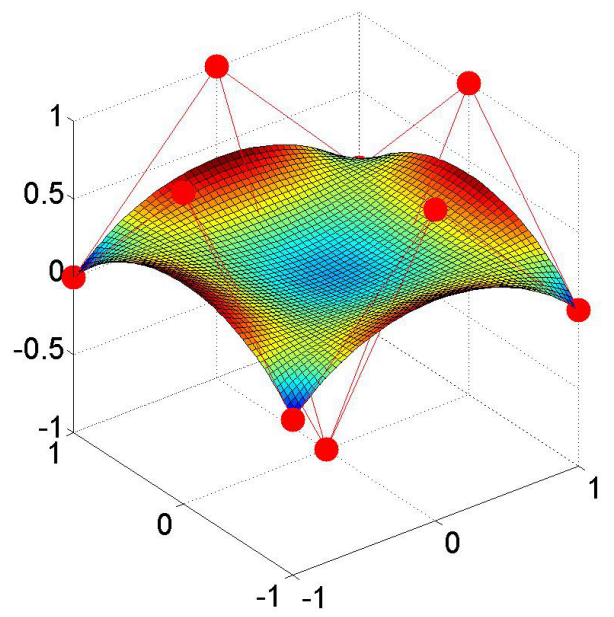

$(b)$

Figure 4. The graphs of $(a)$ the quadratic NURBS surface and $(b)$ the biquadratic NURBS one.

Finally, we show the performances of quadratic NURBS to exactly construct quadric surfaces and model objects. For this purpose we need to consider the spline spaces with unequally smoothness defined in Section 2.4.

In Fig. 5(a) we present the circular cylinder obtained by the control points and weights given in Table 2 and knot vectors $\bar{\xi}=\left\{0, \frac{1}{4}, \frac{1}{2}, \frac{3}{4}, 1\right\}$ (considering the knot $\frac{1}{2}$ with multiplicity two), $\bar{\eta}=\{0,1\}$. In Fig. $5(b)$ we present a goblet obtained by the control points and weights given in Table 3 and knot vectors $\bar{\xi}=\left\{0, \frac{1}{4}, \frac{1}{2}, \frac{3}{4}, 1\right\}$ (considering all the interior knots with multiplicity two), $\bar{\eta}=\left\{0, \frac{1}{5}, \frac{2}{5}, \frac{3}{5}, \frac{4}{5}, 1\right\}$. In such examples, we can observe two different ways of reproducing circles (see also [31, Chap. 7]).

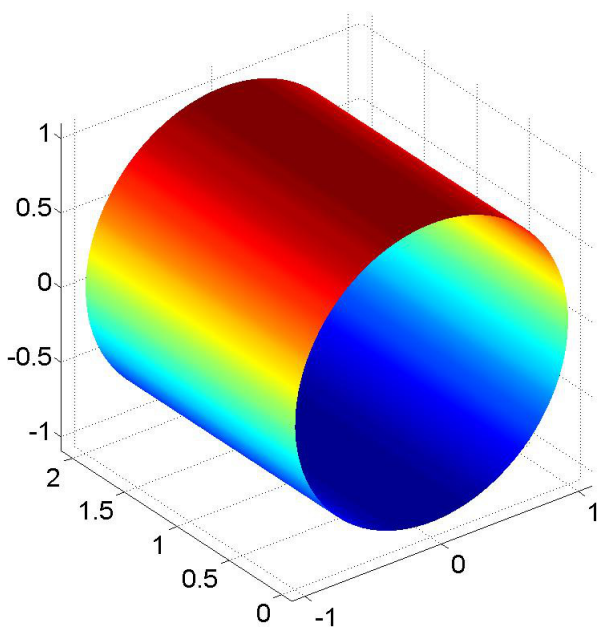

(a)

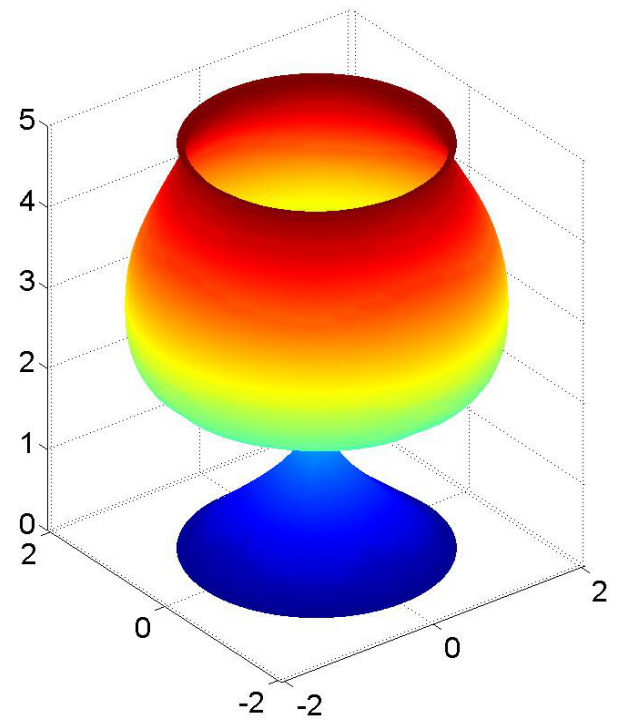

(b)

Figure 5. The quadratic NURBS surfaces reproducing $(a)$ the circular cylinder and $(b)$ the goblet. 
Table 2. Control points and weights of the circular cylinder.

\begin{tabular}{|c|ccc|ccc|}
\hline$i$ & $\mathbf{P}_{i 0}$ & $\mathbf{P}_{i 1}$ & $\mathbf{P}_{i 2}$ & $w_{i 0}$ & $w_{i 1}$ & $w_{i 2}$ \\
\hline 0 & $(0,0,1)$ & $(0,1,1)$ & $(0,2,1)$ & 1 & 1 & 1 \\
1 & $(1,0,1)$ & $(1,1,1)$ & $(1,2,1)$ & $\frac{1}{2}$ & $\frac{1}{2}$ & $\frac{1}{2}$ \\
2 & $(1,0,-1)$ & $(1,1,-1)$ & $(1,2,-1)$ & $\frac{1}{2}$ & $\frac{1}{2}$ & $\frac{1}{2}$ \\
3 & $(0,0,-1)$ & $(0,1,-1)$ & $(0,2,-1)$ & 1 & 1 & 1 \\
4 & $(-1,0,-1)$ & $(-1,1,-1)$ & $(-1,2,-1)$ & $\frac{1}{2}$ & $\frac{1}{2}$ & $\frac{1}{2}$ \\
5 & $(-1,0,1)$ & $(-1,1,1)$ & $(-1,2,1)$ & $\frac{1}{2}$ & $\frac{1}{2}$ & $\frac{1}{2}$ \\
6 & $(0,0,1)$ & $(0,1,1)$ & $(0,2,1)$ & 1 & 1 & 1 \\
\hline
\end{tabular}

Table 3. Control points of the goblet. The weights are $w_{0, j}=w_{2, j}=w_{4, j}=w_{6, j}=w_{8, j}=1, w_{1, j}=w_{3, j}=$ $w_{5, j}=w_{7, j}=\frac{\sqrt{2}}{2}, j=0, \ldots 6$.

\begin{tabular}{|c|cccc|}
\hline$i$ & $\mathbf{P}_{i 0}$ & $\mathbf{P}_{i 1}$ & $\mathbf{P}_{i 2}$ & $\mathbf{P}_{i 3}$ \\
\hline 0 & $(1.5,0,0)$ & $(0.1,0,1)$ & $(0.1,0,2.2)$ & $(2,0,2)$ \\
1 & $(1.5,-1.5,0)$ & $(0.1,-0.1,1)$ & $(0.1,-0.1,2.2)$ & $(2,-2,2)$ \\
2 & $(0,-1.5,0)$ & $(0,-0.1,1)$ & $(0,-0.1,2.2)$ & $(0,-2,2)$ \\
3 & $(-1.5,-1.5,0)$ & $(-0.1,-0.1,1)$ & $(-0.1,-0.1,2.2)$ & $(-2,-2,2)$ \\
4 & $(-1.5,0,0)$ & $(-0.1,0,1)$ & $(-0.1,0,2.2)$ & $(-2,0,2)$ \\
5 & $(-1.5,1.5,0)$ & $(-0.1,0.1,1)$ & $(-0.1,0.1,2.2)$ & $(-2,2,2)$ \\
6 & $(0,1.5,0)$ & $(0,0.1,1)$ & $(0,0.1,2.2)$ & $(0,2,2)$ \\
7 & $(1.5,1.5,0)$ & $(0.1,0.1,1)$ & $(0.1,0.1,2.2)$ & $(2,2,2)$ \\
8 & $(1.5,0,0)$ & $(0.1,0,1)$ & $(0.1,0,2.2)$ & $(2,0,2)$ \\
\hline \hline$i$ & $\mathbf{P}_{i 4}$ & $\mathbf{P}_{i 5}$ & $\mathbf{P}_{i 6}$ & \\
\hline 0 & $(2.1,0,3.7)$ & $(1.2,0,5)$ & $(1.5,0,5)$ & \\
1 & $(2.1,-2.1,3.7)$ & $(1.2,-1.5,5)$ & $(1.5,-1.5,5)$ & \\
2 & $(0,-2.1,3.7)$ & $(0,-1.2,5)$ & $(0,-1.5,5)$ & \\
3 & $(-2.1,-2.1,3.7)$ & $(-1.2,-1.2,5)$ & $(-1.5,-1.5,5)$ & \\
4 & $(-2.1,0,3.7)$ & $(-1.2,0,5)$ & $(-1.5,0,5)$ & \\
5 & $(-2.1,2.1,3.7)$ & $(-1.2,1.2,5)$ & $(-1.5,1.5,5)$ & \\
6 & $(0,2.1,3.7)$ & $(0,1.2,5)$ & $(0,1.5,5)$ & \\
7 & $(2.1,2.1,3.7)$ & $(1.2,1.2,5)$ & $(1.5,1.5,5)$ & \\
8 & $(2.1,0,3.7)$ & $(1.2,0,5)$ & $(1.5,0,5)$ & \\
\hline
\end{tabular}




\section{Applications to the Numerical Solution of Diffusion Problems}

In this section, we propose applications of the NURBS presented in Section 3 to the numerical solution of diffusion problems with mixed boundary conditions. We remark that the proposed method can be extended to more general second order elliptic problems.

Let $\Omega \subset \mathbb{R}^{2}$ be an open, bounded and Lipschitz domain, whose boundary $\partial \Omega$ is partitioned into two relatively open subsets, $\Gamma_{D}$ and $\Gamma_{N}$, i.e. $\emptyset \subseteq \Gamma_{D}, \Gamma_{N} \subseteq \partial \Omega, \Gamma_{D} \neq \emptyset, \Gamma_{D} \cap \Gamma_{N}=\emptyset$ and $\partial \Omega=\bar{\Gamma}_{D} \cup \bar{\Gamma}_{N}$. We consider the problem

$$
\begin{cases}-\nabla \cdot(K \nabla u)=f, & \text { in } \Omega, \\ u=g, & \text { on } \Gamma_{D},(\text { Dirichlet condition }) \\ \frac{\partial u}{\partial \mathbf{n}_{K}}=g_{N}, & \text { on } \Gamma_{N}, \text { (Neumann condition) }\end{cases}
$$

where $K \in \mathbb{R}^{2 \times 2}$ is a symmetric positive-definite matrix, $\mathbf{n}_{K}=K \mathbf{n}$ is the outward conormal vector on $\Gamma_{N}, f \in L^{2}(\Omega), g_{N} \in L^{2}\left(\Gamma_{N}\right)$ and $g \in H^{1 / 2}\left(\Gamma_{D}\right)$, having denoted by $H^{1 / 2}\left(\Gamma_{D}\right)$ the space of functions of $L^{2}\left(\Gamma_{D}\right)$ that are traces of functions of $H^{1}(\Omega)$, with $H^{1}(\Omega):=\left\{v \in L^{2}(\Omega): D^{\alpha} v \in L^{2}(\Omega),|\alpha| \leq 1\right\}$.

In our approach, the problem (11) is solved using the Galerkin procedure.

First of all, assuming as reference parametric domain $\Omega_{0}=[0,1] \times[0,1]$, the physical domain $\Omega$ is described by a global geometry function $\mathbf{G}: \Omega_{0} \rightarrow \bar{\Omega}$. Since many domains of interest in engineering problems are often described by conic sections, we consider as geometry function $\mathbf{G}$ a quadratic NURBS surface on a criss-cross triangulation, presented in Section 3, in order to exactly reproduce them. More precisely, we consider a criss-cross triangulation $\mathcal{T}_{m n}$ of $\Omega_{0}$, the space $\mathcal{S}_{2}^{1}\left(\mathcal{T}_{m n}\right)$, its spanning set $\mathcal{B}_{m n}$ and, given opportune weights $\left\{w_{i j}\right\}_{(i, j) \in \mathcal{K}_{m n}}$, from $\mathcal{B}_{m n}$ we get $\mathcal{N}_{m n}$ defined in $(9)$. Then, we define the global geometry function

$$
\mathbf{G}: \Omega_{0} \rightarrow \bar{\Omega}, \quad \mathbf{G}(\xi, \eta)=\left(\begin{array}{l}
x \\
y
\end{array}\right)
$$

with

$$
\mathbf{G}(\xi, \eta)=\sum_{(i, j) \in \mathcal{K}_{m n}} \mathbf{Q}_{i j} R_{i j}(\xi, \eta), \quad \mathbf{Q}_{i j} \in \mathbb{R}^{2}
$$

We remark that to construct the surface $\mathbf{G}$ in (13), we use all the functions in the spanning set $\mathcal{N}_{m n}$. In this case, the surface (13) has both the convex hull property and the affine transformation invariance one.

In order to explain our approach in details, we first assume homogeneous Dirichlet boundary conditions, i.e. $g \equiv 0$ in (11). The classical weak formulation of (11) is to find $u \in H_{\Gamma_{D}}^{1}:=\left\{v \in H^{1}(\Omega): v=0\right.$ on $\left.\Gamma_{D}\right\}$, such that

$$
a(u, v)=F(v), \quad \forall v \in H_{\Gamma_{D}}^{1}
$$

where $a(\cdot, \cdot)$ is of the continuous bilinear form $a(u, v):=\int_{\Omega}(K \nabla u) \cdot \nabla v d \Omega$ defined in $H_{\Gamma_{D}}^{1} \times H_{\Gamma_{D}}^{1}$ and $F$ is of the linear continuous form $F(v):=\int_{\Omega} f v d \Omega+\int_{\Gamma_{N}} g_{N} v d \Gamma_{N}$ defined in $H_{\Gamma_{D}}^{1}$.

The well known Galerkin method to approximate the solution of (14) consists in selecting a family of finite dimensional subspaces $\mathcal{V}_{h} \subset H^{1}(\Omega)$ depending on a positive parameter $h>0$, and finding $u_{h}$ $\in \mathcal{V}_{D_{h}}$, such that

$$
a\left(u_{h}, v_{h}\right)=F\left(v_{h}\right), \quad \forall v_{h} \in \mathcal{V}_{D_{h}},
$$

where $\mathcal{V}_{D_{h}}:=\left\{v_{h} \in \mathcal{V}_{h}: v_{h}=0\right.$ on $\left.\Gamma_{D}\right\}$

The choice of the space $\mathcal{V}_{h}$ is crucial and the main difference between the existing methods consists in the selection of such a space. In this paper, we assume

$$
\mathcal{V}_{h}=\left\{v_{h} \in H^{1}(\Omega): v_{h}=v_{0, h} \circ \mathbf{G}^{-1}, v_{0, h} \in \mathcal{S}_{2}^{1}\left(\mathcal{T}_{m n}\right)\right\}
$$

and the basis functions of $\mathcal{V}_{h}$ are the compositions of the basis functions of $\mathcal{S}_{2}^{1}\left(\mathcal{T}_{m n}\right)$ with the inverse of G.

We recall that, in practical applications, the geometry of $\Omega$ is frequently described on a mesh of relatively few elements, while the computation of an approximate solution to the problem is performed 
on a refined mesh (fine enough to achieve the desired accuracy). Therefore, we assume that there is a coarsest mesh, of which all the other meshes are a refinement, and that the description of the geometry is fixed at the level of the coarsest mesh and it remains unchanged, independent of the refinement required by the accuracy of the approximation. Then, we introduce refined discrete spaces in order to get accurate numerical solutions, performing the $h$-refinement. This kind of refinement can be done without affecting the geometry, and can be implemented by knot insertion [8].

Furthermore, as explained in Section 1, we treat independently the geometry and the discrete space, since the computation with B-spline spaces is easily implemented and strictly related to NURBS. Indeed, we consider suitable NURBS surfaces based on bivariate quadratic B-splines on criss-cross triangulations, in order to reproduce the physical domain and we use the same above B-splines as the basis for the solution space. In this way, we keep a unique description of the geometry, while avoiding the use of rational functions in the discretization of the solution.

We consider the B-spline basis $\mathcal{B}$ of $\mathcal{S}_{2}^{1}\left(\mathcal{T}_{m n}\right)$, given in $(5)$. For the sake of simplicity, we arrange the functions in $\mathcal{B}$ by using only one index and we denote them by $\Phi_{l}, l=1, \ldots N_{h}$, where $N_{h}$ is the dimension of $\mathcal{S}_{2}^{1}\left(\mathcal{T}_{m n}\right)$, given in (1).

Then, we can define a basis for $\mathcal{V}_{h}$ as

$$
\left\{\varphi_{l}(x, y)=\Phi_{l} \circ \mathbf{G}^{-1}(x, y), l=1, \ldots N_{h}\right\} .
$$

Moreover, let $\left\{\varphi_{l}, l=1, \ldots n_{h}\right\}, n_{h} \leq N_{h}$, be the set of the basis functions of $\mathcal{V}_{h}$ which vanish at $\Gamma_{D}$, then the approximate solution $u_{h}$ of the problem (15) is given by

$$
u_{h}=\sum_{l=1}^{n_{h}} q_{l} \varphi_{l}, \quad q_{l} \in \mathbb{R} .
$$

So, the weak formulation (15) gives rise to the linear system $A \mathbf{q}=\mathbf{f}$ where

- $A \in \mathbb{R}^{n_{h} \times n_{h}}$ is a symmetric, positive definite matrix, called stiffness matrix, with elements

$$
A_{k l}=a\left(\varphi_{l}, \varphi_{k}\right)=\int_{\Omega}\left(K \nabla \varphi_{l}\right) \cdot \nabla \varphi_{k} d \Omega, \quad k, l=1, \ldots, n_{h}
$$

- $\mathbf{f} \in \mathbb{R}^{n_{h}}$ is the vector with components

$$
\mathbf{f}_{k}=F\left(\varphi_{k}\right)=\int_{\Omega} f \varphi_{k} d \Omega+\int_{\Gamma_{N}} g_{N} \varphi_{k} d \Gamma_{N}=\mathbf{f}_{k}^{(1)}+\mathbf{f}_{k}^{(2)}, \quad k=1, \ldots, n_{h}
$$

- $\mathbf{q} \in \mathbb{R}^{n_{h}}$ is the vector of the unknown coefficients $q_{l}$ of $(16), l=1, \ldots, n_{h}$.

The integrals $A_{k l}$ in (17) and $\mathbf{f}_{k}^{(1)}$ in (18), can be transformed as follows

$$
\begin{gathered}
A_{k l}=\int_{\Omega_{0}}\left(K\left[J^{-T} \nabla \Phi_{l}\right]\right) \cdot\left[J^{-T} \nabla \Phi_{k}\right]|\operatorname{det} J| d \Omega_{0}, \quad k, l=1, \ldots, n_{h}, \\
\mathbf{f}_{k}^{(1)}=\int_{\Omega_{0}}(f \circ \mathbf{G}) \Phi_{k}|\operatorname{det} J| d \Omega_{0}, \quad k=1, \ldots, n_{h},
\end{gathered}
$$

with $J$ the Jacobian matrix of the parametrization $\mathbf{G}$ given in (12) and (13).

To evaluate the boundary term $\mathbf{f}_{k}^{(2)}$ in (18), we first define the map $\mathbf{G}_{b}: I:=(0,1) \rightarrow \Gamma_{N}$ as the restriction of $\mathbf{G}$ to the subset of $\partial \Omega_{0}$ mapped into $\Gamma_{N}$, assuming that each side of $\Omega_{0}$ is completely mapped into $\Gamma_{N}$ or $\Gamma_{D}$. Then,

$$
\mathbf{f}_{k}^{(2)}=\int_{I}\left(g_{N} \circ \mathbf{G}_{b}\right) \Phi_{k}\left|\mathbf{G}_{b}^{\prime}\right| d I
$$

For the evaluation of the integrals in (19), (20), we use a composite Gaussian cubature on triangular domains (see [32]), implemented by the Matlab function triquad ${ }^{2}$. Given in input an integer $p$ and the

\footnotetext{
2 von Winckel, G., Matlab procedure triquad,

http://www.mathworks.com/matlabcentral/fileexchange/9230-gaussian-quadrature-for-triangles.
} 
vertices of a triangle of $\mathcal{T}_{m n}$, this procedure computes the $p^{2}$ nodes and the corresponding weights of the rule, whose precision degree is $2 p-1$. In the numerical tests proposed in the sequel, we use $p=2$. When $\mathbf{G}$ is the identity map (i.e. $\bar{\Omega} \equiv \Omega_{0}$ ), then the integral in (19) is exactly computed, since in each triangle of $\mathcal{T}_{m n}$ the integrand function is a bivariate quadratic polynomial.

In order to evaluate $\nabla \Phi_{k}$ and $J$ at the cubature nodes in (19), (20), we compute the values of the B-spline derivatives by means of their BB-coefficients $[6,30]$ and we apply the de Casteljau algorithm for triangular surfaces.

To evaluate the integral in (21), we use a classical composite Gaussian rule with precision degree three, inherited from the one defined in the whole domain.

In the non homogeneous case, i.e. $g \neq 0$ in (11), it is necessary to assume $g \in H^{1 / 2}\left(\Gamma_{D}\right)$, so there exists a function $R_{g} \in H^{1}(\Omega)$ with $\left.R_{g}\right|_{\Gamma_{D}}=g$. We suppose $R_{g}$ is known. Now we set $\stackrel{\circ}{u}=u-R_{g}$ and we notice that it belongs to $H_{\Gamma_{D}}^{1}$. Then, the weak formulation of (11) may be transformed into finding $\stackrel{\circ}{u} \in H_{\Gamma_{D}}^{1}$, such that

$$
a(\stackrel{\circ}{u}, v)=F(v), \quad \forall v \in H_{\Gamma_{D}}^{1},
$$

where $a(\cdot, \cdot)$ is of the bilinear form appearing in (14) and the linear functional $F$ now takes the form

$$
F(v):=\int_{\Omega} f v d \Omega+\int_{\Gamma_{N}} g_{N} v d \Gamma_{N}-\int_{\Omega}\left(K \nabla R_{g}\right) \cdot \nabla v d \Omega .
$$

In order to use the Galerkin method for (22), we need to construct an approximation of $R_{g}$ in $\mathcal{V}_{h}$. The first step is to obtain an approximation $g_{h}$ of the boundary function $g$. A possible approach is to consider the space $\mathcal{V}_{h} \backslash \mathcal{V}_{D_{h}}=\left\langle\varphi_{n_{h}+1}, \ldots, \varphi_{n_{h}+n_{h}^{b}}\right\rangle$, with $n_{h}+n_{h}^{b}=N_{h}$ and $g_{h}$ as a combination of the $n_{h}^{b}$ basis functions of $\mathcal{V}_{h}$ which do not vanish on $\Gamma_{D}$, restricted to $\Gamma_{D}$, i.e.

$$
g_{h}(x, y)=\left.\sum_{l=n_{h}+1}^{n_{h}+n_{h}^{b}} q_{l} \varphi_{l}\right|_{\Gamma_{D}}(x, y), \quad \forall(x, y) \in \Gamma_{D}, \quad q_{l} \in \mathbb{R} .
$$

The control variables $q_{l}$ have to be computed in order to satisfy the prescribed Dirichlet boundary conditions. Here we apply one of the univariate schemes proposed in Section 2.3 to the function $g \circ \mathbf{G}$. Then, the approximation of $R_{g}$ in $\mathcal{V}_{h}$ is the function $R g_{h}(x, y)=\sum_{l=n_{h}+1}^{n_{h}+n_{h}^{b}} q_{l} \varphi_{l}(x, y),(x, y) \in \Omega$.

Now, we compute $\stackrel{\circ}{u} \in_{h} \mathcal{V}_{D_{h}}$ such that

$$
a\left(\stackrel{\circ}{h}_{h}, v_{h}\right)=F\left(v_{h}\right) \quad \forall v_{h} \in \mathcal{V}_{D_{h}} .
$$

The weak formulation (25) leads to a linear system of the form $A \mathbf{q}=\mathbf{f}$, where, in this case, the vector $\mathbf{f}$ is defined according to (23). The approximate solution of (11) will then be provided by $u_{h}=\stackrel{\circ}{u_{h}}+R g_{h}$.

\subsection{Numerical Tests}

Now, we propose some numerical tests to show the performance of our method, with comparisons with other classical methods based on finite elements and/or tensor product $C^{1}$ biquadratic B-splines. Such results have been obtained by procedures implemented in the Matlab environment.

We first exactly reproduce the physical domain by the global geometry function (13), constructed specifying the knot partitions $\bar{\xi}, \bar{\eta}$, the control points and the weights. This initial exact representation is retained during the refinement process. Then, we perform $h$-refinement by adding at every step a middle knot in each interval of the partitions $\bar{\xi}$ and $\bar{\eta}$.

For each refinement, we construct the approximation $g_{h}$, defined in (24), by the three spline schemes proposed in Section 2.3. Finally, we consider the Galerkin discretization, we solve the corresponding linear system and we obtain the approximate solution $u_{h}$. 
We compute the discrete $L^{\infty}$-norm of the error $\left(u-u_{h}\right)$ on a $100 \times 100$ grid of evaluation points, uniform in $\Omega_{0}$. Following the notations of Section 2.3, we denote by $\mathbf{E}$ (i), $\mathbf{E}$ (ii) and $\mathbf{E}$ (iii) the error norms obtained in computing the control variables in (24) by the three corresponding schemes (i), (ii) and (iii). We also compute the $H^{1}$-norm of the error $\left(u-u_{h}\right)$, by the composite Gaussian cubature on triangular domains (see [32]) already used in Section 4 . We denote by $\mathbf{E}^{1}$ (i), $\mathbf{E}^{1}$ (ii) and $\mathbf{E}^{1}$ (iii) the error norms obtained in computing the control variables in (24) by the three corresponding schemes (i), (ii) and (iii).

With reference to the condition number $\kappa_{2}(A)=\|A\|_{2}\left\|A^{-1}\right\|_{2}$, we remark that its growth is controlled with respect to the order of the matrix $A$, since we have verified that its behaviour is linear with respect to the size of $A$.

Example 1 We consider a Poisson problem in a quarter of an annulus, shown in Fig. 6(b), with mixed non homogeneous boundary conditions

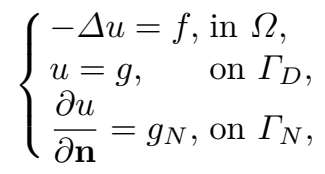

where

$$
\begin{aligned}
& \Omega=\left\{(x, y): x=r \cos (\theta), y=r \sin (\theta), r \in(1,4), \theta \in\left(\frac{\pi}{2}, \pi\right)\right\}, \\
& \Gamma_{D}=\{(0, y): y \in[1,4]\} \cup\{(x, 0): x \in[-4,-1]\}, \\
& \Gamma_{N}=\left\{(\cos (\theta), \sin (\theta)): \theta \in\left[\frac{\pi}{2}, \pi\right]\right\} \cup\left\{(4 \cos (\theta), 4 \sin (\theta)): \theta \in\left[\frac{\pi}{2}, \pi\right]\right\}
\end{aligned}
$$

and $g, g_{N}$ are obtained from the exact solution

$$
u(x, y)=\sin \left(\frac{x^{2}+y^{2}-1}{5}\right) .
$$

In order to exactly reproduce the domain, we consider the knot partitions $\bar{\xi}=\bar{\eta}=\{0,1\}$ (see Fig. 6(a)). Therefore, we have $\mathcal{K}_{11}=\{(i, j): 0 \leq i, j \leq 2\}$ and

$$
\mathbf{G}(\xi, \eta)=\sum_{(i, j) \in \mathcal{K}_{11}} \mathbf{P}_{i j} R_{i j}(\xi, \eta), \quad(\xi, \eta) \in \Omega_{0},
$$

with control points and weights given in Table 4 [31, Chap. 7].

Table 4. Control points and weights of the geometry function in Example 1.

\begin{tabular}{|c|ccc|ccc|}
\hline$i$ & $\mathbf{P}_{i 0}$ & $\mathbf{P}_{i 1}$ & $\mathbf{P}_{i 2}$ & $w_{i 0}$ & $w_{i 1}$ & $w_{i 2}$ \\
\hline 0 & $(-1,0)$ & $(-1,1)$ & $(0,1)$ & 1 & $\frac{\sqrt{2}}{2}$ & 1 \\
1 & $(-2.5,0)$ & $(-2.5,2.5)$ & $(0,2.5)$ & 1 & 1 & 1 \\
2 & $(-4,0)$ & $(-4,4)$ & $(0,4)$ & 1 & $\frac{\sqrt{2}}{2}$ & 1 \\
\hline
\end{tabular}

Then, we perform $h$-refinement, considering $m, n=2,4,8,16,32$ and, in Figs. 7 and 8 , we report the graphs of the error $L^{\infty}$-norms and of the error $H^{1}$-norms, respectively, versus the interval number per side $m, n$. We can notice that the numerical convergence order computed by $\mathbf{E}$ (i) is near-optimal, instead with the schemes (ii) and (iii) we achieve the optimal convergence order for the $L^{\infty}$-norm. In the case of $H^{1}$-norm we have the optimal convergence order 2 for all the three schemes (i), (ii) and (iii).

Moreover, in Fig. 7 we also present the graph of the $L^{\infty}$-norm of the errors computed with the same $h$-refinement by classical linear FEM, denoted by $\mathbf{E} \mathbb{P}_{1}$, by quadratic FEM, denoted by $\mathbf{E} \mathbb{P}_{2}$ and by classical $C^{1}$ biquadratic B-splines, denoted by $\mathbf{E} S_{2,2}^{1,1}$.

We can notice that our results outperform those obtained by classical linear and quadratic FEM and are comparable with those obtained by classical $C^{1}$ biquadratic B-splines. Moreover, quadratic FEM loses one order of convergence, due to the geometry approximation influence.

In Fig. 9(a) we give the graph of the exact solution (26) and in Fig. 9(b) the graph of the approximate solution, computed by our method, with $m=n=8$ and the scheme (iii), presented in Section 2.3. 


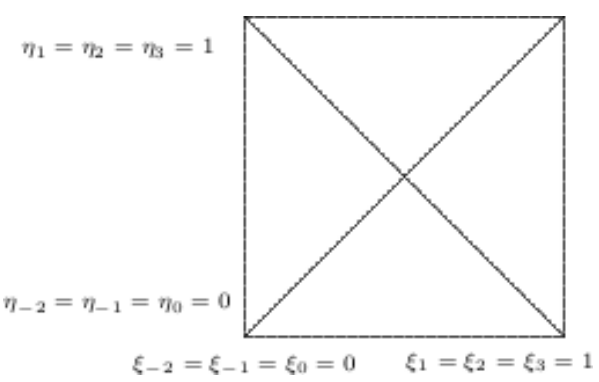

(a)

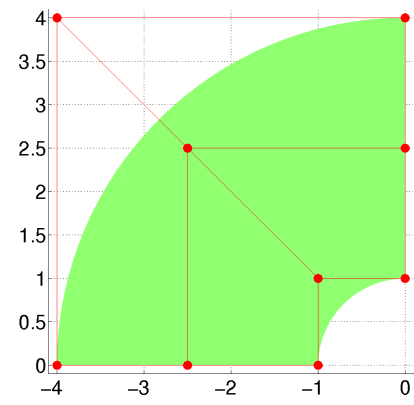

(b)

Figure 6. (a) Parameter domain $\Omega_{0}$ and (b) physical domain $\Omega$ of Example 1 .

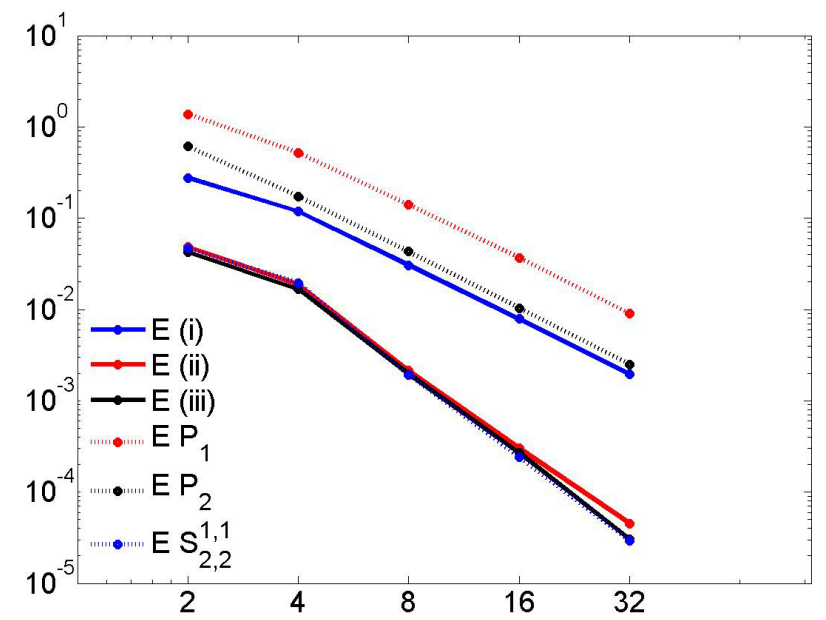

Figure 7. Comparisons of the $L^{\infty}$-norm of the errors versus interval number per side in Example 1.

Example 2 Now, we consider the Poisson problem with Dirichlet conditions in the unit square

$$
\begin{cases}-\Delta u=f, & \text { in } \Omega=(0,1)^{2}, \\ u=g, & \text { on } \partial \Omega,\end{cases}
$$

where $f$ and $g$ are obtained from the exact solution $u(x, y)=\frac{1}{6}\left(x^{3}+y^{3}\right)+(x+y-1)|x+y-1|$. We can notice that the function $u$ is a function with a discontinuity of the second directional derivative in the direction $\left[\frac{\sqrt{2}}{2}, \frac{\sqrt{2}}{2}\right]^{T}$ across the line $x+y-1=0$. We present this example in order to show the different behaviour of the approximated solutions in the spaces $\mathcal{S}_{2}^{1}\left(\mathcal{T}_{m n}\right)$ and $\mathcal{S}_{2,2}^{1,1}\left(\mathcal{R}_{m n}\right)$.

In order to exactly reproduce the domain, we consider the knot partitions $\bar{\xi}=\bar{\eta}=\{0,1\}$. Therefore, we have $\mathcal{K}_{11}=\{(i, j): 0 \leq i, j \leq 2\}$ and

$$
\mathbf{G}(\xi, \eta)=\sum_{(i, j) \in \mathcal{K}_{11}} \mathbf{P}_{i j} B_{i j}(\xi, \eta), \quad(\xi, \eta) \in \Omega_{0} .
$$

Since $\mathbf{G}$ is the identity map, the control points are the nine points $\mathbf{P}_{i j}=\left\{\left(s_{i}, t_{j}\right), 0 \leq i, j \leq 2\right\}$, where $s_{i}$ and $t_{j}$ are defined in (6). Obviously, in the case of $\mathcal{S}_{2,2}^{1,1}\left(\mathcal{R}_{m n}\right)$ the map $\mathbf{G}$ is described by the same control points and knot partitions, but the blending functions are the biquadratic B-splines on $\bar{\xi} \times \bar{\eta}$.

Then, we perform $h$-refinement, considering $m, n=2,4,8,16,32$ and, in Tables 5 and 6 , we report the error $L^{\infty}$-norms and the error $H^{1}$-norms, versus the interval number per side $m, n$, for the two above spline spaces $\mathcal{S}_{2}^{1}\left(\mathcal{T}_{m n}\right)$ and $\mathcal{S}_{2,2}^{1,1}\left(\mathcal{R}_{m n}\right)$, respectively. For the space $\mathcal{S}_{2,2}^{1,1}\left(\mathcal{R}_{m n}\right)$, we denote the error $L^{\infty}$-norms by $\mathbf{E}_{t p}$ (i), $\mathbf{E}_{t p}$ (ii), $\mathbf{E}_{t p}$ (iii), and the error $H^{1}$-norms by $\mathbf{E}_{t p}^{1}$ (i), $\mathbf{E}_{t p}^{1}$ (ii), $\mathbf{E}_{t p}^{1}$ (iii). 


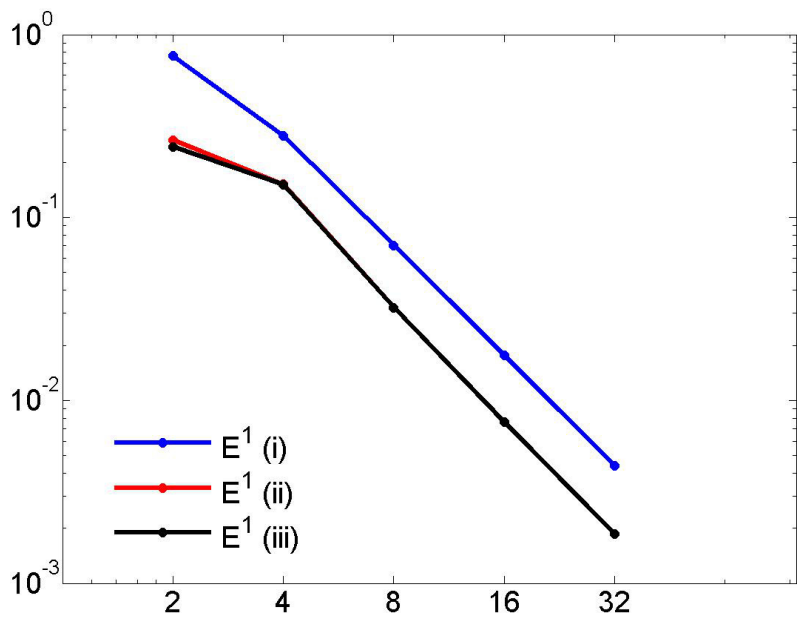

Figure 8. Comparisons of the $H^{1}$-norm of the errors versus interval number per side in Example 1.

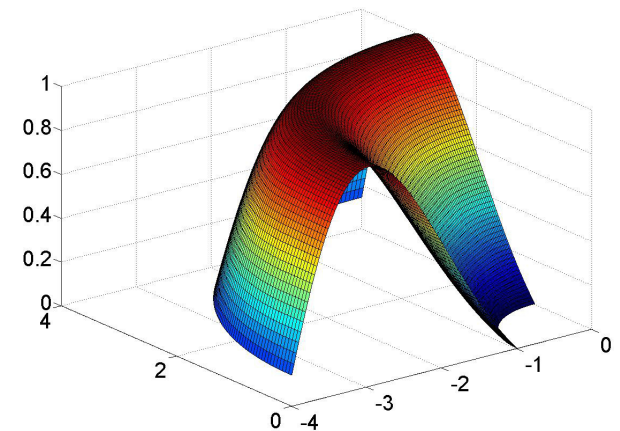

(a)

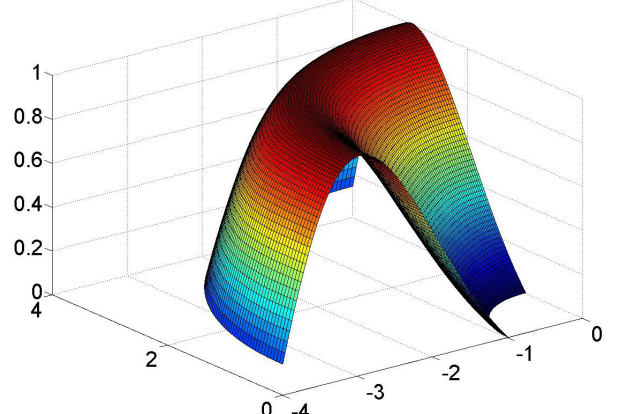

(b)

Figure 9. The graphs of $(a)$ the exact solution and $(b)$ the approximate solution of Example 1.

Table 5. Comparisons of the $L^{\infty}$-norm and the $H^{1}$-norm of the errors versus interval number per side in Example 2 for the space $\mathcal{S}_{2}^{1}\left(\mathcal{T}_{m n}\right)$.

\begin{tabular}{|c||c|c|c||c|c|c|}
\hline$m=n$ & $\mathbf{E}(\mathbf{i})$ & $\mathbf{E}(\mathbf{i i})$ & $\mathbf{E}(\mathbf{i i i})$ & $\mathbf{E}^{1}(\mathbf{i})$ & $\mathbf{E}^{1}(\mathbf{i i})$ & $\mathbf{E}^{1}(\mathbf{i i i})$ \\
\hline 2 & $7.8(-02)$ & $3.6(-03)$ & $2.0(-03)$ & $1.8(-01)$ & $1.4(-02)$ & $1.2(-02)$ \\
4 & $2.1(-02)$ & $4.6(-04)$ & $2.5(-04)$ & $5.3(-02)$ & $3.2(-03)$ & $3.1(-03)$ \\
8 & $5.6(-03)$ & $5.7(-05)$ & $3.1(-05)$ & $1.5(-02)$ & $7.7(-04)$ & $7.6(-04)$ \\
16 & $1.4(-03)$ & $6.8(-06)$ & $3.9(-06)$ & $3.9(-03)$ & $1.9(-04)$ & $1.9(-04)$ \\
32 & $3.6(-04)$ & $8.5(-07)$ & $4.9(-07)$ & $1.0(-03)$ & $4.8(-05)$ & $4.8(-05)$ \\
\hline
\end{tabular}

Table 6. Comparisons of the $L^{\infty}$-norm and the $H^{1}$-norm of the errors versus interval number per side in Example 2 for the space $\mathcal{S}_{2,2}^{1,1}\left(\mathcal{R}_{m n}\right)$.

\begin{tabular}{|c||c|c|c||c|c|c|}
\hline$m=n$ & $\mathbf{E}_{t p}(\mathbf{i})$ & $\mathbf{E}_{t p}(\mathbf{i i})$ & $\mathbf{E}_{t p}(\mathbf{i i i})$ & $\mathbf{E}_{t p}^{1}(\mathbf{i})$ & $\mathbf{E}_{t p}^{1}(\mathbf{i i})$ & $\mathbf{E}_{t p}^{1}(\mathbf{i i i})$ \\
\hline 2 & $1.1(-01)$ & $1.1(-01)$ & $1.1(-01)$ & $3.6(-01)$ & $3.0(-01)$ & $3.0(-01)$ \\
4 & $2.3(-02)$ & $2.4(-02)$ & $2.4(-02)$ & $1.1(-01)$ & $9.3(-02)$ & $9.3(-02)$ \\
8 & $1.9(-02)$ & $1.7(-02)$ & $1.7(-02)$ & $5.1(-02)$ & $4.8(-02)$ & $4.8(-02)$ \\
16 & $1.1(-02)$ & $1.1(-02)$ & $1.1(-02)$ & $2.8(-02)$ & $2.7(-02)$ & $2.7(-02)$ \\
32 & $3.5(-03)$ & $3.5(-03)$ & $3.5(-03)$ & $1.0(-02)$ & $9.6(-03)$ & $9.6(-03)$ \\
\hline
\end{tabular}

We can remark that the numerical results obtained by $C^{1}$ quadratic splines on criss-cross triangulations outperform those obtained by $C^{1}$ biquadratic splines. Indeed the first ones reproduce the discontinuity of 
the second directional derivative across $x+y-1=0$ of the exact solution $u$, unlike the approximated solutions in $\mathcal{S}_{2,2}^{1,1}\left(\mathcal{R}_{m n}\right)$.

Finally, in Fig. 10(a) we give the graph of the exact solution and in Fig. 10(b) the graph of its first directional derivative in the direction $\left[\frac{\sqrt{2}}{2}, \frac{\sqrt{2}}{2}\right]^{T}$. Moreover, in Figs. 11(a), 12(a) and 11(b), 12(b) we report the graphs of the approximate solutions and the corresponding first directional derivatives, computed with $m=n=8$ and the scheme (iii) of Section 2.3, in the space $\mathcal{S}_{2}^{1}\left(\mathcal{T}_{88}\right)$ and in the space $\mathcal{S}_{2,2}^{1,1}\left(\mathcal{R}_{88}\right)$, respectively.

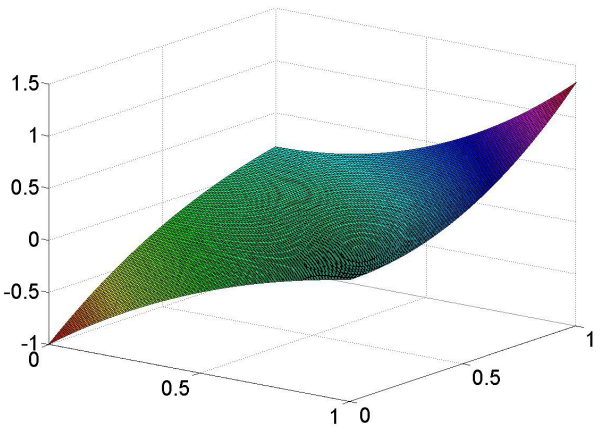

(a)

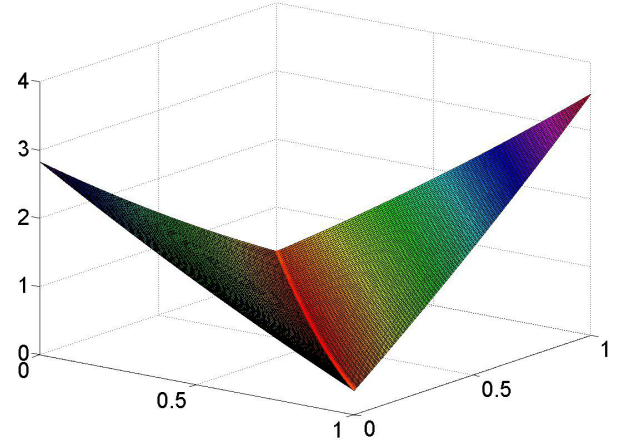

$(b)$

Figure 10. The graphs of $(a)$ the exact solution and $(b)$ its first directional derivative in the direction $\left[\frac{\sqrt{2}}{2}, \frac{\sqrt{2}}{2}\right]^{T}$ of Example 2.

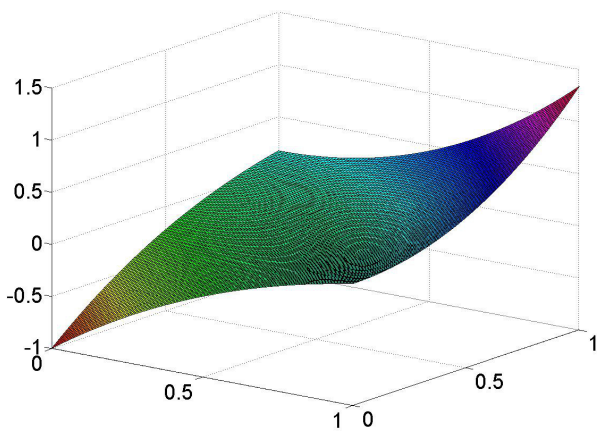

(a)

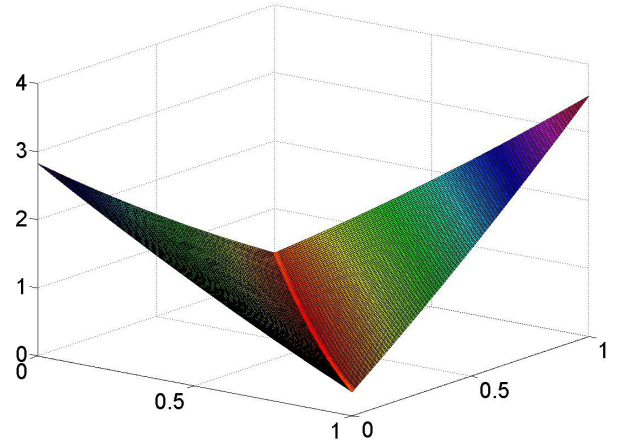

(b)

Figure 11. The graphs of $(a)$ the approximate solution in the space $\mathcal{S}_{2}^{1}\left(\mathcal{T}_{88}\right)$ and $(b)$ the corresponding first directional derivative in the direction $\left[\frac{\sqrt{2}}{2}, \frac{\sqrt{2}}{2}\right]^{T}$.

We can note that the first directional derivative of the approximate solution in the space $\mathcal{S}_{2}^{1}\left(\mathcal{T}_{m n}\right)$ is $C^{0}$ across the line $x+y-1=0$ (see Fig. 11(b)), confirming the properties of such a spline space. Indeed, as already remarked, spline functions of total degree defined on criss-cross triangulations allow the control of smoothness across directions not only parallel to the coordinate axes, as it occurs in the case of tensor product splines (see Fig. 12(b)). Moreover, we can notice unwanted oscillations in the first directional derivative of the approximate solution belonging to $\mathcal{S}_{2,2}^{1,1}\left(\mathcal{T}_{88}\right)$ (see Fig. 12(b)).

Example 3 Finally, we present a very simple example to show how the different schemes in the choice of the control variables in (24), influence the approximation of the solution. We consider the Poisson 


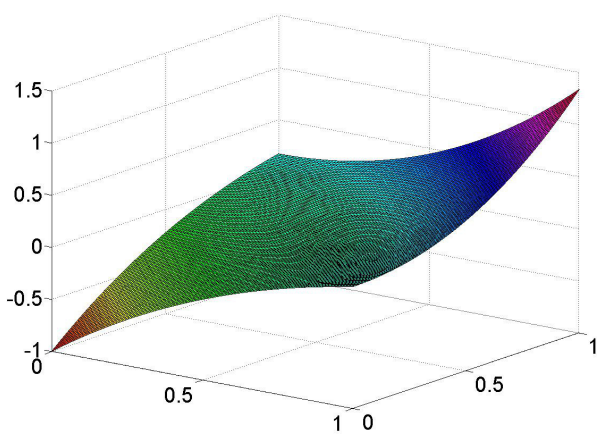

(a)

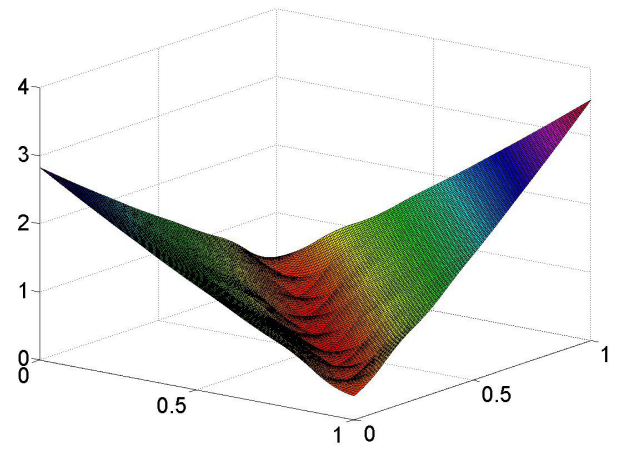

$(b)$

Figure 12. The graphs of $(a)$ the approximate solution in the space $\mathcal{S}_{2,2}^{1,1}\left(\mathcal{R}_{88}\right)$ and $(b)$ the corresponding first directional derivative in the direction $\left[\frac{\sqrt{2}}{2}, \frac{\sqrt{2}}{2}\right]^{T}$.

problem in the unit square

$$
\left\{\begin{array}{l}
-\Delta u=f, \text { in } \Omega=(0,1)^{2}, \\
u=g, \quad \text { on } x=0, y=0 \\
\frac{\partial u}{\partial \mathbf{n}}=g_{N}, \text { on } x=1, y=1,
\end{array}\right.
$$

where $f, g$ and $g_{N}$ are obtained from the exact solution $u(x, y)=x^{2}+y^{2}$. In order to exactly reproduce the domain, we consider the same map $\mathbf{G}$ of Example 2.

Then, we perform $h$-refinement, considering $m, n=2,4,8,16,32$ and, in Table 7 , we report the error $L^{\infty}$-norms and the error $H^{1}$-norms, versus the interval number per side $m, n$.

Table 7. Comparisons of the $L^{\infty}$-norm and the $H^{1}$-norm of the errors versus interval number per side in Example 3 .

\begin{tabular}{|c||c|c|c||c|c|c|}
\hline$m=n$ & $\mathbf{E}(\mathbf{i})$ & $\mathbf{E}(\mathbf{i i})$ & $\mathbf{E}(\mathbf{i i i})$ & $\mathbf{E}^{1}(\mathbf{i})$ & $\mathbf{E}^{1}(\mathbf{i i})$ & $\mathbf{E}^{1}(\mathbf{i i i})$ \\
\hline 2 & $6.2(-02)$ & $4.7(-15)$ & $4.0(-15)$ & $9.0(-02)$ & $3.5(-15)$ & $3.5(-15)$ \\
4 & $1.6(-02)$ & $3.3(-15)$ & $3.3(-15)$ & $2.5(-02)$ & $3.0(-15)$ & $3.0(-15)$ \\
8 & $3.9(-03)$ & $4.4(-15)$ & $4.4(-15)$ & $6.3(-03)$ & $4.4(-15)$ & $4.3(-15)$ \\
16 & $9.8(-04)$ & $4.2(-15)$ & $4.4(-15)$ & $1.6(-03)$ & $8.9(-15)$ & $8.5(-15)$ \\
32 & $2.4(-04)$ & $1.6(-14)$ & $1.6(-14)$ & $4.0(-04)$ & $2.3(-14)$ & $2.3(-14)$ \\
\hline
\end{tabular}

We can notice the solution, which is a quadratic polynomial, is reproduced by the schemes (ii) and (iii) proposed in Section 2.3, thanks to their optimal approximation properties. Moreover, the computation of derivatives and integrals is stable, because there is not deterioration of the approximation error with the increase of the refinement.

\section{$5 \quad$ Final Remarks}

In this paper we have considered and analysed NURBS based on bivariate quadratic B-splines on criss-cross triangulations of the parametric domain $\Omega_{0}=[0,1] \times[0,1]$, presenting their main properties and showing their performances to exactly construct quadric surfaces and model objects. Moreover, we have proposed applications to the numerical solution of partial differential equations, with mixed boundary conditions on a given physical domain $\Omega$, by using three different spline methods to set the prescribed Dirichlet boundary conditions. Finally, we have provided numerical tests.

We remark that the proposed method can be extended to more general second order elliptic problems. Moreover, an interesting open problem can be the definition and the study of hierarchical bivariate 
quadratic spline functions on criss-cross triangulations, enabling to local refinement and their application to the solution of differential problems, also in the context of Isogeometric Analysis. Finally, we recall that the extension of the proposed scheme to the trivariate setting by using spline spaces of total degree is an interesting open problem. Such spaces are defined on complex 3D partitions, as for example tetrahedral or prismatic ones (see e.g. [7,26,33,34,35,36]).

Acknowledgments. The authors thank the University of Torino for its support to their research.

\section{References}

1. R. Wang and C. Li, "A kind of multivariate NURBS surfaces," J. Comp. Math., vol. 22, pp. 137-144, 2004.

2. C. K. Chui, L. L. Schumaker, and R. H. Wang, "On spaces of piecewise polynomials with boundary conditions III. Type-2 triangulations," J. Can. Math. Soc., Conf. Proc., vol. 3, pp. 67-80, 1983.

3. R. Wang and T. He, "The spline spaces with boundary conditions on nonuniform type-2 triangulation," Kexue Tongbao, vol. 30, no. 7, pp. 858-861, 1985.

4. R. Wang, Multivariate Spline Functions and Their Applications. Science Press, Beijing/New York, Kluwer Academic Publishers, Dordrecht/Boston/London, 2001.

5. P. Sablonnière, "BB-coefficients of basic quadratic splines on rectangular domains with uniform criss-cross triangulations," Prépublication 02-56, INSA \& IRMAR, Rennes, 2002.

6. —— "Quadratic B-splines on non-uniform criss-cross triangulations of bounded rectangular domains of the plane," Prépublication 03-14, INSA \& IRMAR, Rennes, 2003.

7. — "Quadratic spline quasi-interpolants on bounded domains of $\mathbb{R}^{d}, d=1,2,3$," Rend. Sem. Mat. Univ. Pol. Torino, vol. 61, pp. 229-246, 2003.

8. — - "Refinement equation and subdivision algorithm for quadratic B-splines on non-uniform criss-cross triangulations," in Wavelets and splines. St. Petersburg Univ. Press, 2005, pp. 84-102.

9. C. Dagnino, S. Remogna, and P. Sablonnière, "Error bounds on the approximation of functions and partial derivatives by quadratic spline quasi-interpolants on non-uniform criss-cross triangulations of a rectangular domain," BIT Numerical Mathematics, vol. 53, pp. 87-109, 2013.

10. E. B. Ameur, D. Barrera Rosillo, and D. Sbibih, "Subdivision scheme of quartic bivariate splines on a four-directional mesh," in ESAIM: PROCEEDINGS, 20, 2007, pp. 16-28.

11. D. Barrera-Rosillo and M. J. Ibáñez Pérez, "On a class of spline discrete quasi-interpolants," in Curve and Surface Fitting: Avignon 2006, Albert Cohen, Jean-Louis Merrien, and Larry L. Schumaker (eds.). Nashboro Press, Brentwood, 2007, pp. 21-30.

12. A. Abbadi, D. Barrera, M. J. Ibáñez, and D. Sbibih, "A general method for constructing quasi-interpolants from B-splines," Journal of Computational and Applied Mathematics, vol. 234, pp. 1324-1337, 2010.

13. D. Barrera, A. Guessab, M. J. Ibáñez, and O. Nouisser, "Optimal bivariate $C^{1}$ cubic quasi-interpolation on a type-2 triangulation," Journal of Computational and Applied Mathematics, vol. 234, pp. 1188-1199, 2010.

14. D. Barrera, M. J. Ibáñez, P. Sablonnière, and D. Sbibih, "On near-best discrete quasi-interpolation on a four-directional mesh," Journal of Computational and Applied Mathematics, vol. 233, pp. 1470-1477, 2010.

15. D. Barrera, A. Guessab, M. J. Ibáñez, and O. Nouisser, "Construction techniques for multivariate modified quasi-interpolants with high approximation order," Computers and Mathematics with Applications, vol. 65, pp. 29-41, 2013.

16. — " "Increasing the approximation order of spline quasi-interpolants," Journal of Computational and Applied Mathematics, vol. 252, pp. 27-39, 2013.

17. C. Allouch, P. Sablonnière, and D. Sbibih, "A collocation method for the numerical solution of a two dimensional integral equation using a quadratic spline quasi-interpolant," Numer. Algorithms, vol. 62, pp. 445-468, 2013.

18. P. Lamberti, "Numerical integration based on bivariate quadratic spline quasi-interpolants on bounded domains," BIT Numerical Mathematics, vol. 49, pp. 565-588, 2009.

19. C. Dagnino and P. Lamberti, "On the construction of local quadratic spline quasi-interpolants on bounded rectangular domains," Journal of Computational and Applied Mathematics, vol. 221, pp. 367-375, 2008.

20. F. Foucher and P. Sablonnière, "Approximating partial derivatives of first and second order by quadratic spline quasi-interpolants on uniform meshes," Math. Comput. Simulation, vol. 77, pp. 202-208, 2008.

21. D. Barrera and M. J. Ibáñez, "Minimizing the quasi-interpolation error for bivariate discrete quasi-interpolants," Journal of Computational and Applied Mathematics, vol. 224, pp. 250-268, 2009.

22. C. Dagnino, P. Lamberti, and S. Remogna, "Curve network interpolation by $C^{1}$ quadratic B-spline surfaces," Computer Aided Geometric Design, vol. 40, pp. 26-39, 2015. 
23. C. Dagnino and P. Sablonnière, "Error analysis for quadratic spline quasi-interpolants on non-uniform criss-cross triangulations of bounded rectangular domains," Prépublication 06-06, INSA É IRMAR, Rennes, 2006.

24. C. Dagnino and P. Lamberti, "Spline "quasi-interpolants" with boundary conditions on criss-cross triangulations," in Curve and Surface Fitting: Avignon 2006, Albert Cohen, Jean-Louis Merrien, and Larry L. Schumaker (eds.). Nashboro Press, Brentwood, 2007, pp. 101-110.

25. C. de Boor, K. Höllig, and S. D. Riemenschneider, Box Splines. Springer, 1993.

26. M. J. Lai and L. L. Schumaker, Spline functions on triangulations. Cambridge University Press, 2007.

27. P. Sablonnière, "Bernstein-Bézier methods for the construction of bivariate spline approximants," Computer Aided Geometric Design, vol. 2, pp. 29-36, 1985.

28. C. de Boor, A practical guide to splines (Revised edition). Springer, 2001.

29. C. Dagnino, P. Lamberti, and S. Remogna, "B-spline bases for unequally smooth quadratic spline spaces on non-uniform criss-cross triangulations," Numer. Algorithms, vol. 61, pp. 209-222, 2012.

30. — - "BB-coefficients of unequally smooth quadratic B-splines on non uniform criss-cross triangulations," Quaderni Scientifici del Dipartimento di Matematica n. 24, Università di Torino, http://hdl.handle.net/2318/434, 2008.

31. L. Piegl and W. Tiller, The NURBS Book. Springer, 1995.

32. J. N. Lyness and R. Cools, "A survey of numerical cubature over triangles," Mathematics and Computer Science Division, Argonne National Laboratory, III, 1994.

33. S. Remogna, "Quasi-interpolation operators based on the trivariate seven-direction $C^{2}$ quartic box spline," BIT Numerical Mathematics, vol. 51, pp. 757-776, 2011.

34. S. Remogna and P. Sablonnière, "On trivariate blending sums of univariate and bivariate quadratic spline quasi-interpolants on bounded domains," Computer Aided Geometric Design, vol. 28, pp. 89-101, 2011.

35. C. Dagnino, P. Lamberti, and S. Remogna, "Numerical integration based on trivariate $C^{2}$ quartic spline quasi-interpolants," BIT Numerical Mathematics, vol. 53, pp. 873-896, 2013.

36. - "Near-best $C^{2}$ quartic spline quasi-interpolants on type-6 tetrahedral partitions of bounded domains," CALCOLO, vol. 52, pp. 475-494, 2015. 